\title{
Transient Scattering from Two Cavities Embedded in a Ground Plane
}

\author{
Richard Uber and Aihua Wood \\ Department of Mathematics and Statistics \\ Air Force Institute of Technology \\ Wright-Patterson AFB, OH 45419, USA \\ richard.uber@afit.edu
}

\begin{abstract}
This work presents a numerical method for modeling electromagnetic scattering from two-dimensional cavities embedded in a perfectly conducting ground plane. First, the problem is discretized in time with Newmark's method, then solved using finite-element boundary-integral technique. A proof is presented to demonstrate that the discretized variational formulation is well-posed at each time step.
\end{abstract}

\section{INTRODUCTION}

Electromagnetic scattering from open cavities and cavitybacked apertures has been an active area of research with applications in aircraft design and radar cross-section (RCS) modeling. Past work on cavity scatterers has either focused on single-cavity environments, as in [4], [2], or the time-harmonic domain, as in [1], [3]. Transient applications are increasingly in demand because they may be applied wide-band and nonlinear problems. This paper consolidates previous work by Van, Li, and Wood [4], [3] by presenting an application of the Newmark method to discretize and solve a two-cavity scattering problem. Additionally, it is proved that the formulation at each time step is well-posed.

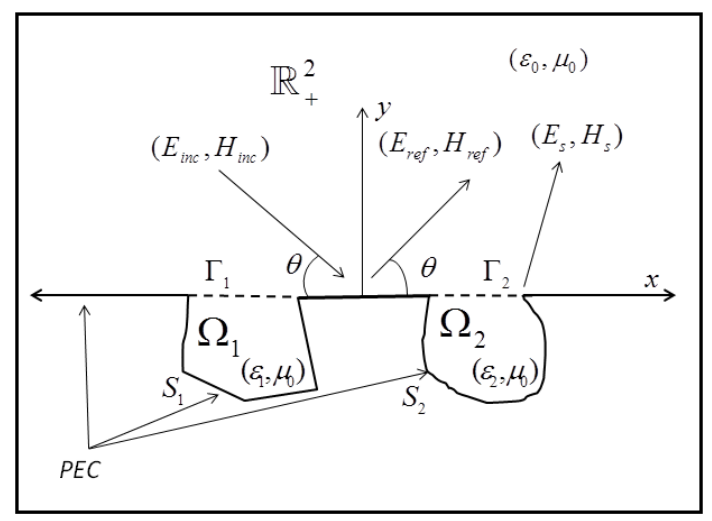

Fig. 1. Two cavity environment

\section{Problem Statement}

\section{A. Problem Environment}

This work considers an infinite perfectly-conducting ground plane (PEC) with two fully-embedded two-dimensional cavities. In three dimensions, these cavities are $z$-invariant infinite grooves, or channels. The $\mathrm{TM}^{z}$ orientation is considered here, indicating that the electric field will be fully $z$-directed, described as a function of $x$ and $y$. Above the ground plane is considered free space $\mathbb{R}_{+}^{2}=\left\{x, y \in \mathbb{R}^{2}: y \geq 0\right\}$ with homogeneous parameters $\varepsilon_{0}, \mu_{0}$. The cavities, denoted $\Omega_{1}, \Omega_{2}$, may be filled with non-magnetic, homogeneous, dielectric medium, with parameters $\mu_{0}, \varepsilon_{j}, j=1,2 . \varepsilon_{j} \in \mathbb{C}, \operatorname{Re}\left\{\varepsilon_{j}\right\}>$ $0, \operatorname{Im}\left\{\varepsilon_{j}\right\} \geq 0$. PEC cavity walls are denoted $S_{1}, S_{2}$ and cavity apertures denoted $\Gamma_{1}, \Gamma_{2}$, respectively for cavities 1 and 2 . This environment is illustrated in Fig. 1.

\section{B. Transient Scattering Problem}

The problem is posed as an initial value problem. Given an incident $\mathrm{TM}^{z}$ electric field $u^{i n c}(x, y, t)$ defined in $\mathbb{R}_{+}^{2} \times t \geq 0$, find the total field $u(x, y, T)$ for a given time $T>0$ and $(x, y) \in \mathbb{R}_{+}^{2}$ subject to:

$$
\left\{\begin{aligned}
-\Delta u+\varepsilon_{r} \frac{\partial^{2} u}{\partial t^{2}}=0 & \text { in } \mathbb{R}_{+}^{2} \cup \Omega_{1} \cup \Omega_{2}, \\
u=0 & \text { on } \Gamma^{c} \cup S_{1} \cup S_{2}, \\
\lim _{y \rightarrow 0+} \frac{\partial u}{\partial y}=\lim _{y \rightarrow 0-} \frac{\partial u}{\partial y} & \forall x:(x, 0) \in \Gamma_{1} \cup \Gamma_{2},
\end{aligned}\right.
$$

In the upper half plane, $u=u^{i n c}+u^{r e f}+u^{s}$, where $u^{r e f}$ is defined by a full reflection from a PEC ground plane. $u^{s} \in \mathbb{R}_{+}^{2} \times[0, \infty)$ is purely outgoing, and subject to the standard radiation condition. Finally, we restrict the solution space to $\mathrm{H}^{1}\left(\mathbb{R}_{+}^{2} \cup \Omega_{1} \cup \Omega_{2}\right)$ so the differential form of Maxwell's equations are applicable. In the proposed approach, which follows closely the method used in [4], the transient problem is first discretized in time, next the discretized problem is solved using a finite element-boundary integral (FE-BI) method. This process is then iterated using the Newmark method to progress in time.

\section{Newmark Method}

The Newmark method, is applied as in [4]. Using a fixed time step length $h$, the total number of time steps needed to approximate the fields at final time $T$ is $N=T / h$. The notation, $u^{n}(x, y)$ and $\dot{u}^{n}(x, y)$ are used to denote the approximations of $u(x, y, n h)$ and $u_{t}(x, y, n h)$, respectively. For the $\mathrm{TM}^{z}$ case, the generated equations are:

$$
\tilde{u}^{n+1}=u^{n}+h \dot{u}^{n}+\left(\frac{1}{2}-\beta\right) h^{2} \ddot{u}^{n},
$$


which is a prediction based on current time data, and

$$
-\Delta u^{n+1}+\alpha^{2} \varepsilon_{r} u^{n+1}=\alpha^{2} \varepsilon_{r} \tilde{u}^{n+1},
$$

which is of similar form to the time-harmonic wave equation with a source term. Equation (3) is solved for $u^{n+1}$ using the finite element method. Once a solution is obtained, the Newmark correction is applied:

$$
\begin{aligned}
& \ddot{u}^{n+1}=\alpha^{2}\left(u^{n+1}-\tilde{u}^{n+1}\right) \\
& \dot{u}^{n+1}=\dot{u}^{n}+(1-\gamma) h \ddot{u}^{n}+\gamma h \ddot{u}^{n+1}
\end{aligned}
$$

\section{Exterior Solution}

Given that $u^{i n c}$ is known for all time, then by applying image theory, $u^{r e f}$ is also known. That leaves $u^{s}$ as the only unknown in the total exterior field $u$ in $\mathbb{R}_{+}^{2}$. Define $g_{0}$ as $u^{n+1}$ on $\Gamma_{1} \cup \Gamma_{2}$ and zero elsewhere on the ground plane. Given $u^{i n c}$ and $g_{0}$, the exterior fields can be solved analytically using the half-space Green's function, $G_{\alpha}\left(\vec{r}, \overrightarrow{r^{\prime}}\right)$, as demonstrated in [1], [4].

$$
\begin{aligned}
u^{e x t}(\vec{r}) & =u^{i n c}(\vec{r})-u^{i n c}\left(\vec{r}_{i}\right)+u^{s}(\vec{r}) . \\
u^{s}(\vec{r}) & =\alpha^{2} \int_{\mathbb{R}_{+}^{2}} G_{\alpha} \tilde{u}^{s} d \overrightarrow{r^{\prime}}-\int_{\Gamma_{1} \cup \Gamma_{2}} g_{0} \frac{\partial G_{\alpha}}{\partial n} d x^{\prime} . \\
G_{\alpha}\left(\vec{r}, \overrightarrow{r^{\prime}}\right) & =\frac{1}{2 \pi}\left[K_{0}\left(\alpha\left|\vec{r}-\overrightarrow{r^{\prime}}\right|\right)-K_{0}\left(\alpha\left|\vec{r}-\overrightarrow{r_{i}^{\prime}}\right|\right)\right] .
\end{aligned}
$$

Where $\vec{r}=(x, y), \vec{r}_{i}=(x,-y)$, and $K_{0}$ is the modified Bessel function of the second kind. By defining the exterior field in this way, we may construct the Neumann boundary condition for the discretized interior problem.

\section{E. Interior Problem}

The discretized interior problem is posed as a boundary value problem at each time step by combining information from equations (1) and (3). Thus, for each time step, we solve the following boundary value problem:

$$
\begin{cases}-\Delta u^{s}+\alpha^{2} \varepsilon_{r} u^{s}=\alpha^{2} \varepsilon_{r} \tilde{u}^{s} & \text { in } \Omega_{1} \cup \Omega_{2}, \\ \lim _{y \rightarrow 0-} \frac{\partial u}{\partial y}=\lim _{y \rightarrow 0+}\left(2 \frac{\partial u^{i n c}}{\partial y}+\frac{\partial u^{s}}{\partial y}\right) & \text { on } \Gamma_{1} \cup \Gamma_{2}, \\ u=0 & \text { on } S_{1} \cup S_{2} .\end{cases}
$$

After applying Green's theorem and enforcing boundary and radiation conditions, the Neumann boundary condition on $\Gamma_{1} \cup$ $\Gamma_{2}$ can be summarized by:

$$
\left.\frac{\partial u^{n+1}}{\partial y}\right|_{y=0}(x)=2 \frac{\partial u^{i n c, n+1}}{\partial y}(x, 0)+\tilde{H}^{n+1}(x)+\left[T_{\alpha} g\right](x),
$$$$
\tilde{H}^{n+1}(x)=\alpha^{2} \int_{\mathbb{R}_{+}^{2}} \frac{\partial G_{\alpha}}{\partial y}\left((x, 0), \overrightarrow{r^{\prime}}\right) \tilde{u}^{s, n+1}\left(\overrightarrow{r^{\prime}}\right) d \overrightarrow{r^{\prime}},
$$$$
\left[T_{\alpha} g\right](x)=\frac{1}{\pi} \int_{\Gamma_{1} \cup \Gamma 2}\left[\frac{\partial^{2}}{\partial x^{2}}-\alpha^{2}\right] K_{0}\left(\alpha\left|x-x^{\prime}\right|\right) g_{0}\left(x^{\prime}\right) d x^{\prime} .
$$

We employ the finite element method to solve weakly the interior problem expressed in (8), deriving the variational formulation:

$$
a\left(u^{n+1}, v\right)=b^{n+1}(v),
$$

defined by:

$$
\begin{aligned}
a(u, v) & =\langle\nabla u, \nabla v\rangle_{\Omega_{1} \cup \Omega_{2}}+\alpha^{2}\left\langle\varepsilon_{r} u, v\right\rangle_{\Omega_{1} \cup \Omega_{2}}-\langle T u, v\rangle_{\Gamma_{1} \cup \Gamma_{2}}, \\
b(v) & =\alpha^{2}\left\langle\varepsilon_{r} \tilde{u}, v\right\rangle_{\Omega_{1} \cup \Omega_{2}}+\left\langle 2 \frac{\partial u^{i n c}}{\partial y}+\tilde{H}, v\right\rangle_{\Gamma_{1} \cup \Gamma_{2}} .
\end{aligned}
$$

\section{RESUlTS}

The principal result of this work is the proof that the variational formulation (10) is well-posed. This proof builds upon the work in [4] which established that the one-cavity problem, similarly derived, is well-posed. We show that the bilinear operator $a(\cdot, \cdot)$ is coercive in $\mathrm{H}^{1}\left(\Omega_{1} \cup \Omega_{2}\right)$ and conclude by the Lax-Milgram theorem that a unique solution exists at each time step.

Theorem The variational formulation (10) has a unique solution $u$ in $\mathrm{H}^{1}\left(\Omega_{1} \cup \Omega_{2}\right)$ and satisfies $\|u\| \leq C\|g\|$ at time $T>0$.

Numerical implementation of the transient FE-BI method

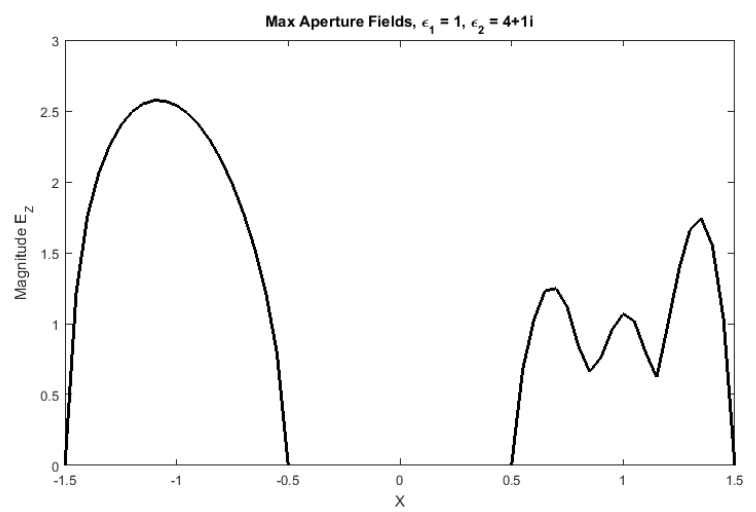

Fig. 2. Continuous Wave Solution

models the transient near-field solution and is consistent with expected results. A sample problem, shown in Fig. 2, shows the maximum aperture fields generated by a continuous wave with wavelength of $1 \mathrm{~m}$.

\section{CONCLUSION}

This work presents a FE-BI method for modeling the transient scattering from two cavities embedded in the ground plane which has a well-posed variational formulation at each time step. This method is expected to be easily extended to multiple cavities embedded in a ground plane. Finally, the FEBI method is in line with current applications used in the industry for cavity-backed apertures.

\section{REFERENCES}

[1] J. M. Jin, The Finite Element Method in Electromagnetics, 3rd ed.. Wiley: Hoboken, NJ, 2014, pp. 381-399.

[2] P. Li, L.L. Wang, and A. W. Wood, "Analysis of transient electromagnetic scattering from a three-dimensional open cavity", SIAM J. Appl. Math., vol. 75, no. 4, pp. 1675-1699, 2015.

[3] P. Li and A. W. Wood, "A two-dimensional Helmholtz equation solution for the multiple cavity scattering problem", J. Comp. Phys., vol. 240, pp. 100-120, 2013.

[4] T. Van and A. W. Wood, "A time-domain finite element method for Helmholtz equations", J. Comp. Phys., vol. 183, pp. 486-507, 2002. 